\title{
Formulation and in vitro evaluation of orally disintegrating film of alprazolam
}

\author{
Zahra Jafariazar', Seyed Kazem Sadjady', Niloufar Chamani ${ }^{3}$, Minoo Afshar $^{2}$ \\ ${ }^{I}$ Associate Professor, Department of Pharmaceutics, Faculty of Pharmacy, Tehran Medical Sciences, Islamic Azad \\ University (IAU), Tehran, Iran \\ ${ }^{2}$ Assistant Professor, Department of Pharmaceutics, Faculty of Pharmacy, Tehran Medical Sciences, Islamic Azad \\ University (IAU), Tehran, Iran \\ ${ }^{3}$ Pharm.D. Student, Department of Pharmaceutics, Faculty of Pharmacy, Tehran Medical Sciences, Islamic Azad \\ University (IAU), Tehran, Iran
}

\begin{abstract}
Background: Over the past years, orally disintegrating films (ODFs) have been a suitable alternative to conventional oral dosage forms such as tablets and capsules, especially for patients with dysphagia. ODF is relatively a new dosage form which is prepared using hydrophilic polymers, which rapidly dissolve on tongue or buccal cavity and has other advantages of rapid onset of action and improved bioavailability. The aim of this study was formulation of ODF dosage forms containing alprazolam, which is a short-acting anxiolytic of the benzodiazepines class of psychoactive drugs.

Materials and methods: Various polymers like hydroxy propyl methyl cellulose (HPMC6cps), sodium carboxy methyl cellulose (Na CMC), polyvinyl alcohol (PVA72000), and different plasticizers like propylene glycol (PG), polyethylene glycol (PEG400) with different percentage using solvent casting technique were used to prepare ODF formulations. Physicochemical tests including weight and thickness determination, disintegration time, assay and dissolution time were performed to find the optimum formulation.

Results: The optimized formulation contained PVA72000 and PEG400 with disintegration time less than 60 seconds and acceptable dissolution rate.

Conclusion: ODT dosage form of alprazolam with acceptable dissolution behavior and proper disintegration time was successfully formulated in this study.
\end{abstract}

Keywords: Alprazolam, Orally disintegrating films, Formulation, In vitro evaluation.

Cited as: Jafariazar Z, Sadjady SK, Chamani N, Afshar M. Formulation and in vitro evaluation of orally disintegrating film of Alprazolam. Medical Science Journal of Islamic Azad University, Tehran Medical Branch 2019; 29(2): 117-124.

Correspondence to: Minoo Afshar

Tel: +982122640052

E-mail: afsharme@sina.tums.ac.ir

ORCID ID: 0000-0002-7209-9893

Received: 20 Nov 2018; Accepted: 4 Dec 2018 
مجله علوم يزشكى دانشعاه آزاد اسلامى

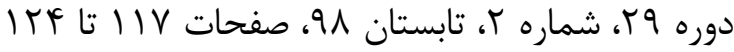

\section{فرمولاسيون و بررسى خصوصيات فيزيكوشيميايى فيلم بازشونده در دهان آلِيرازولام}

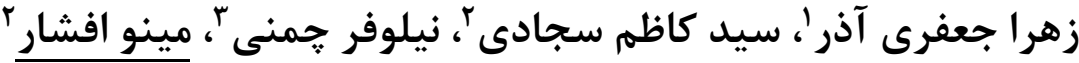

' دانشيار، كروه فارماسيوتيكس، دانشكده داروسازى، علوم يزشكى تهران، دانشكاه آزاد اسلامى، تهران، ايران

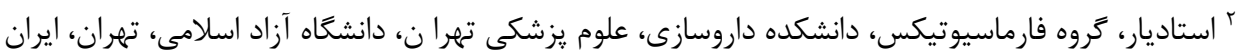

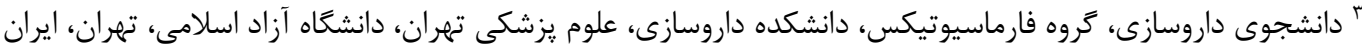

سابقه و هدف: در طول سال هاى كنشته، فيلمهاى سريع بازشونده خوراكى (ODFs) جايكزين مناسبى براى فرمهاى خوراكى سنتى

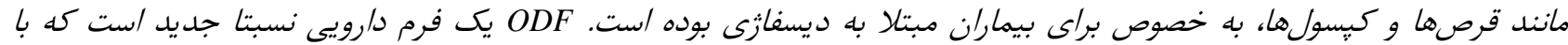

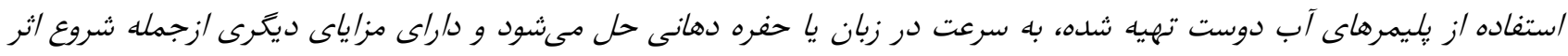

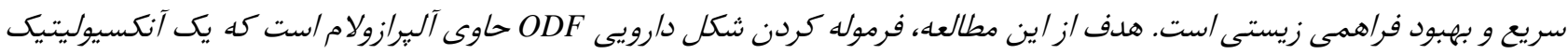

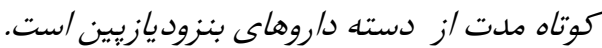
روش بررسى: يليمرهاى مختلف مانند هيدروكسى بروبيل متيل سلولز (HPMC6cps)، سديم كربوكسى متيل سلولز (Na CMC) ، بلى

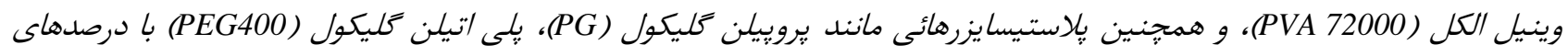

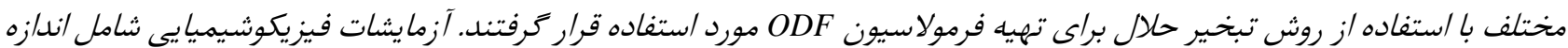

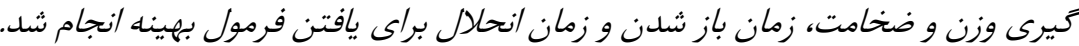
يافتهها: فرمولاسيون بهينه شاهل PVA72000 و PEG400 بود كه زمان باز شلن آن كمتر /ز •و ثانيه بون، ضمن اينكه سرعت حلانليت مناسب د/شت.

نتيجهَيرى: شكل دارويى ODT آليرازولام با رفتار انحلال قابل قبول و زمان بازشدن مناسب با موفقيت در /ين مطالعه فرموله شد. وازگان كليدى: آليرازولام ، فيلم سريع بازشونده دهانى، فرمولاسيون ، خصوصيات فيزيكوشيميايى.

در ميان ساير فرمهاى دارويى به علت يذيرش بالاى بيماران از مقدمه

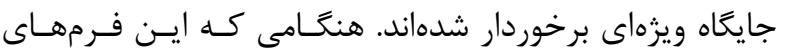

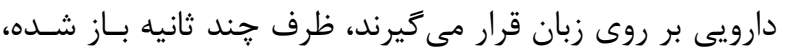

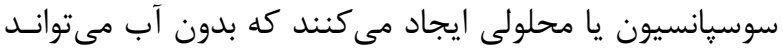

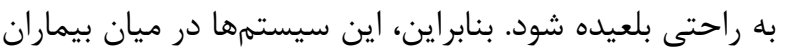
مبتلا به تهوع، ديسفازى، بيمارى حركتى، و اخــتلالات روانى إنى

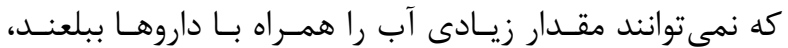

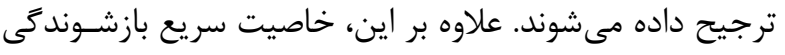

Orally ) بـه تـازگى سيسـتمهــاى سـريع بازشــونده دهــانى مانند قـرصهـاى سـريع بازشـونده (Orally Disintegrating Tablets :ODTs) بازشـونده (ODally Disintegrating Films :ODFs) و ويفرهـا

آدرس نويسنده مسئول: ، علوم يزشكى تهرا ن ، دانشكاه آزاد اسلامى ، كروه فارماسـيوتيكس، مينـو افشـار (email: afsharme@sina.tums.ac.ir) ORCID ID: 0000-0002-7209-9893 9V/N/T9 تاريخ دريافت مقاله تاريخ بذيرش مقاله: 9V/9/IT 
آناليتيكال و ساخت شركت مرك آلمان بودنـد. آب مقطر توسط

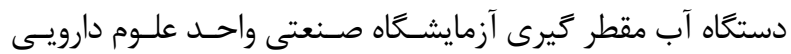
تهيه شد.

تهيه קايههاى يليمرى بدون دارو

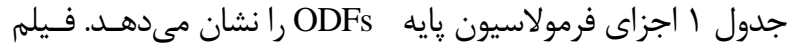

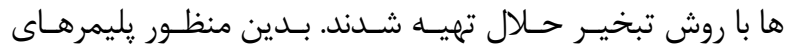

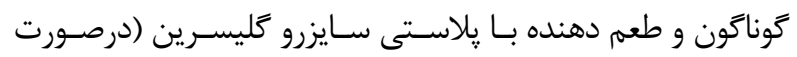

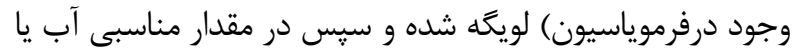

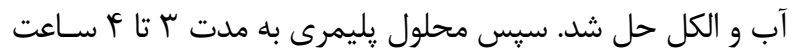

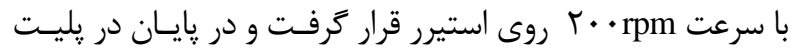

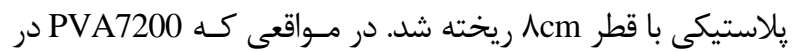

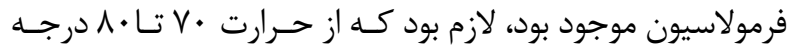

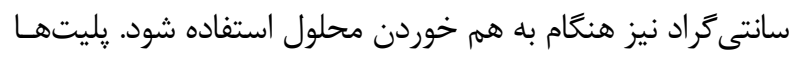

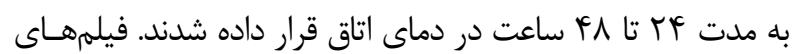

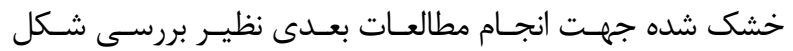

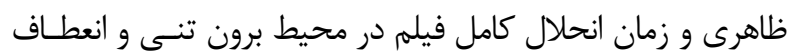

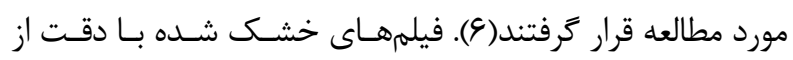

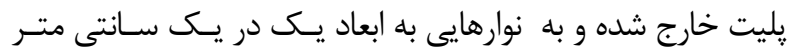
برش داده شدند. فيلمها تا زمـان اسـتفاده در كيسـته يلاسـتيكى إنى دربسته نكمهارى شدند.

\section{تهييه يايههاى يليمرى حاوى دارو نئن}

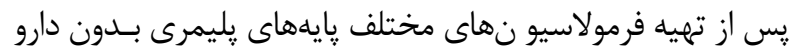

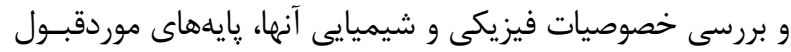

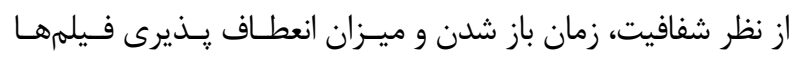

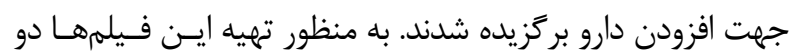

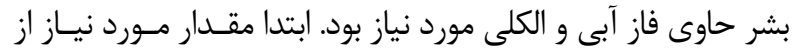

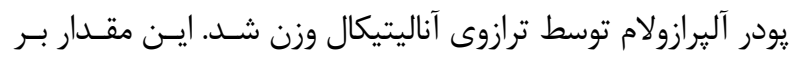

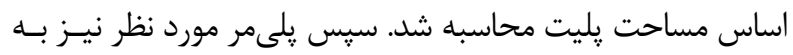

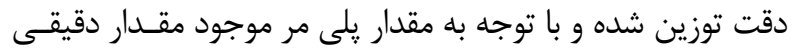

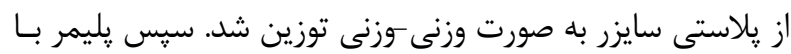

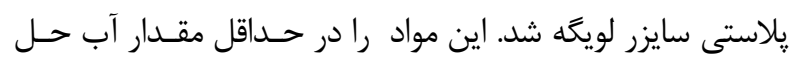

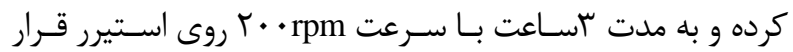
داديم. در مواقعى كه PVA7200 در فرمولاسياسيون موجود بود، لازم

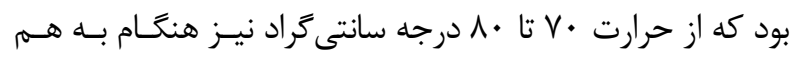

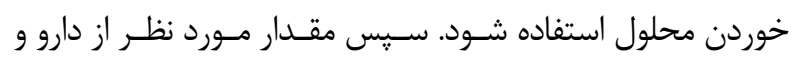

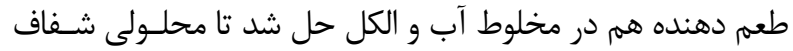
به دست آيد. اين محلول فاز الكلى را تشكيل مى دماد.
آنها باعث افزايش فراهمى زيستى همراه با شروع اثر سـريعتـر دارو در مقايسه با فرمهاى خوراكى معمولى مى شى نود (1).

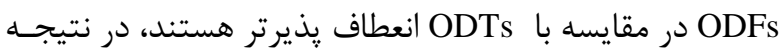

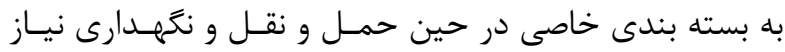

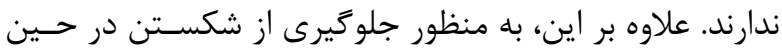

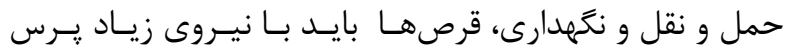

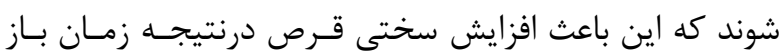

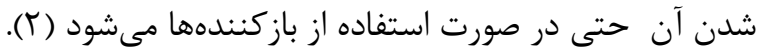

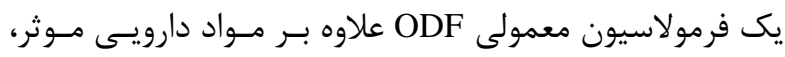

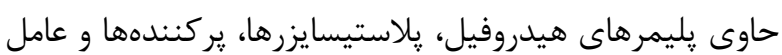

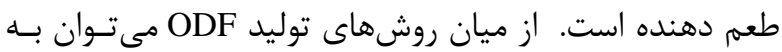

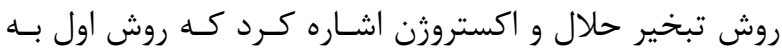

علت سادگى رايجتر است (با).

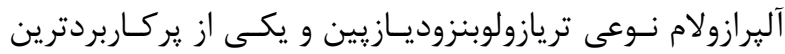

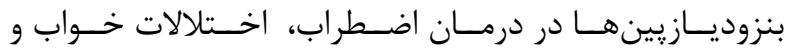

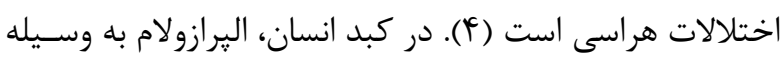

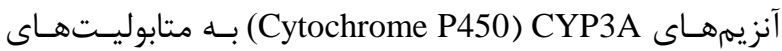
هيدروكسيله متابوليزه مسىشـود. آلفـا هيدروكسى آليــازولام

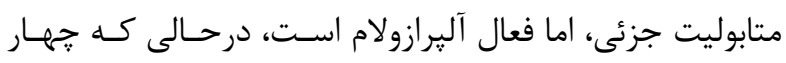

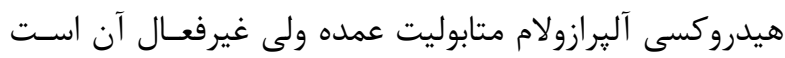

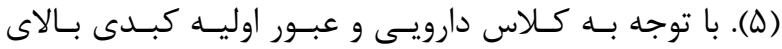

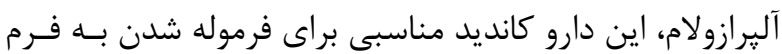

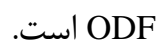
هدف اصلى اين يزوهش ساخت فيلم سريع بازشونده نيم ميلى

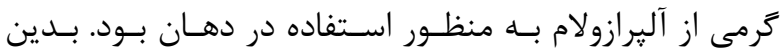

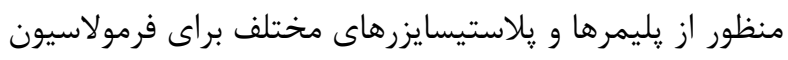

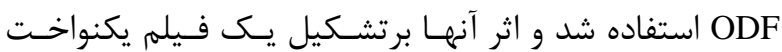
بررسى شد و در پايان آزمايش سرعت حلاليت و تعيين مقـدار

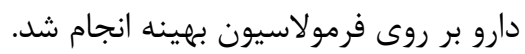

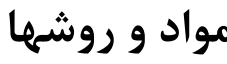

مواد

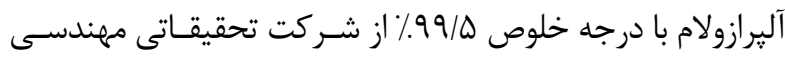

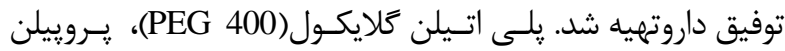

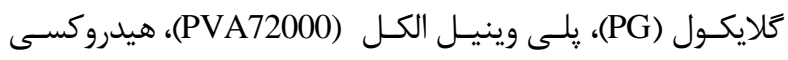

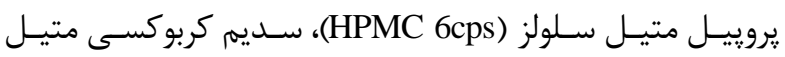

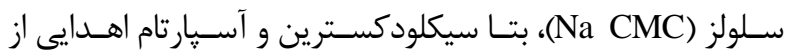

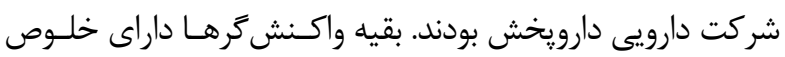




\begin{tabular}{|c|c|c|c|c|c|c|c|}
\hline \multirow[t]{2}{*}{ فرمولاسيون } & \multicolumn{3}{|c|}{ يليمر (ميلى گرم) } & \multicolumn{2}{|c|}{ ״ِلاستيسايزر (ميلى } & \multirow[t]{2}{*}{ گليسرين (ميلى گرم) } & \multirow[t]{2}{*}{ آسيارتام (ميلى ترم) } \\
\hline & HPMC6cps & $\mathrm{Na} \mathrm{MC}$ & PVA7200 & PG & PEG400 & & \\
\hline 1 & TrA & - & - & $f \Delta / 9$ & - & Tr/A & $I T / V$ \\
\hline r & prt & - & - & - & - & $\Lambda \varepsilon / \mathcal{F}$ & $I T / V$ \\
\hline r & FTt & - & - & $\Lambda \varepsilon / T^{4}$ & - & - & $I T / V$ \\
\hline f & FTt & - & - & Fr/T & - & $\Lambda \varepsilon / 4$ & $I T / V$ \\
\hline$\Delta$ & 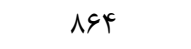 & - & - & $\Lambda \varepsilon / K^{f}$ & - & IVT/A & $I T / V$ \\
\hline 4 & - & RTt & - & Fr/T & - & - & $I T / V$ \\
\hline v & - & GTt & - & Fr/T & - & $\Lambda \varepsilon / \mathcal{F}$ & $I T / V$ \\
\hline$\wedge$ & - & RTt & - & $\Lambda \varepsilon / T^{f}$ & - & - & $I T / V$ \\
\hline 9 & - & GTt & - & $\Lambda \varepsilon / F^{f}$ & - & $1 \cdot 1$ & $I T / V$ \\
\hline 1. & - & $\Lambda \notin F$ & - & $\Lambda \varepsilon / \uparrow^{f}$ & - & - & $I T / V$ \\
\hline 11 & ris & rIS & - & Fr/T & - & $\Lambda \varepsilon / \mathcal{F}$ & $I T / V$ \\
\hline ir & lfF & 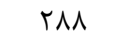 & - & - & - & $1 \% 9 / 9$ & $I T / V$ \\
\hline Ir & rAM & IfF & - & - & - & $1 \% 9 / 9$ & $I T / V$ \\
\hline 14 & $1 \cdot 1$ & MTF & - & - & - & $149 / 9$ & $I T / V$ \\
\hline 10 & - & - & Frt & - & $F r / T$ & - & $I T / V$ \\
\hline 19 & ris & - & TIS & - & Fr/T & - & $I T / V$ \\
\hline IV & IFF & - & $r \Lambda \Lambda$ & - & FT/T & - & $I T / V$ \\
\hline 11 & 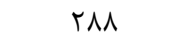 & - & IFF & - & $F r / T$ & - & $I T / V$ \\
\hline 19 & MTF & - & $1 \cdot 1$ & - & $F r / T$ & - & $I T / V$ \\
\hline$r$. & - & rle & ris & - & $F r / T$ & - & $I T / V$ \\
\hline rI & - & $r \wedge \Lambda$ & IfF & - & $\Lambda \varepsilon / \mathcal{F}$ & - & $I T / V$ \\
\hline Tr & - & IFF & 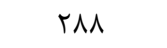 & - & $\Lambda \varepsilon / \mathcal{F}$ & - & $I T / V$ \\
\hline Tr & - & $1 \cdot 1$ & MTF & - & $\Lambda \varepsilon / \mathcal{A}$ & - & $I T / V$ \\
\hline
\end{tabular}

بــه منظــور تنظــيم pH از اســيد ســيتريك اســفاده شــد و فرمولاسيونهايى با pH ه و و 9 تهيه شد.

بررسى تاثير اكسييانتهاى ديگر بر تشكيل فيلم يكنواخت

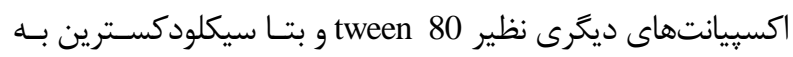

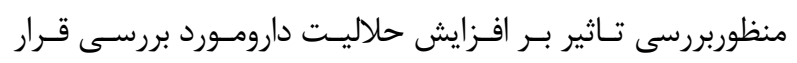

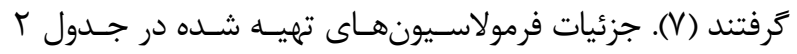
خلاصه شده است.

\section{آزمونهاى كنترل فيزيكوشيميايى}

$$
\text { بررسى خواص ظاهرى فيلم }
$$

جهت انجام اين آزمون، فيلمهاى ساخته شده از يك فرمولاسـيون

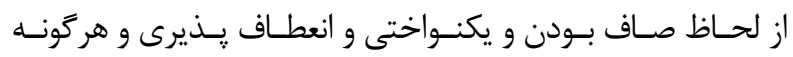
مشكل ظاهرى مورد بررسى قرار گرفتند.
فاز الكلى به صورت سريوشيده به مدت r-r سـاعت روى اسـتيرر

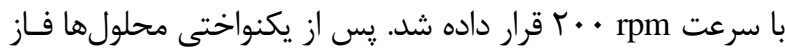
آبى به طور آهسته و قطره قطره به فاز الكلى اضـافه شـد. محلــول حاصل بايد با سرعت بسيار كم به مدت \& تا ها ه ساعت روى استيرر

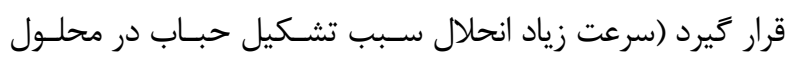

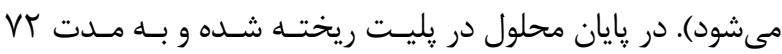

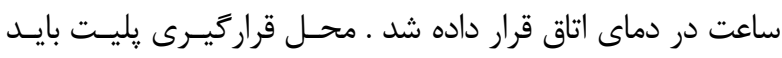

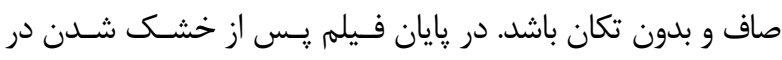

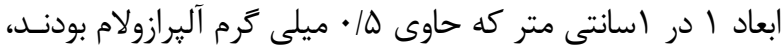
بريده شده و جهت انجام مطالعات بعدى مورد استفاده قرارگرفت. بررسى تاثير pH بر تشكيل فيلم يكنواخت 


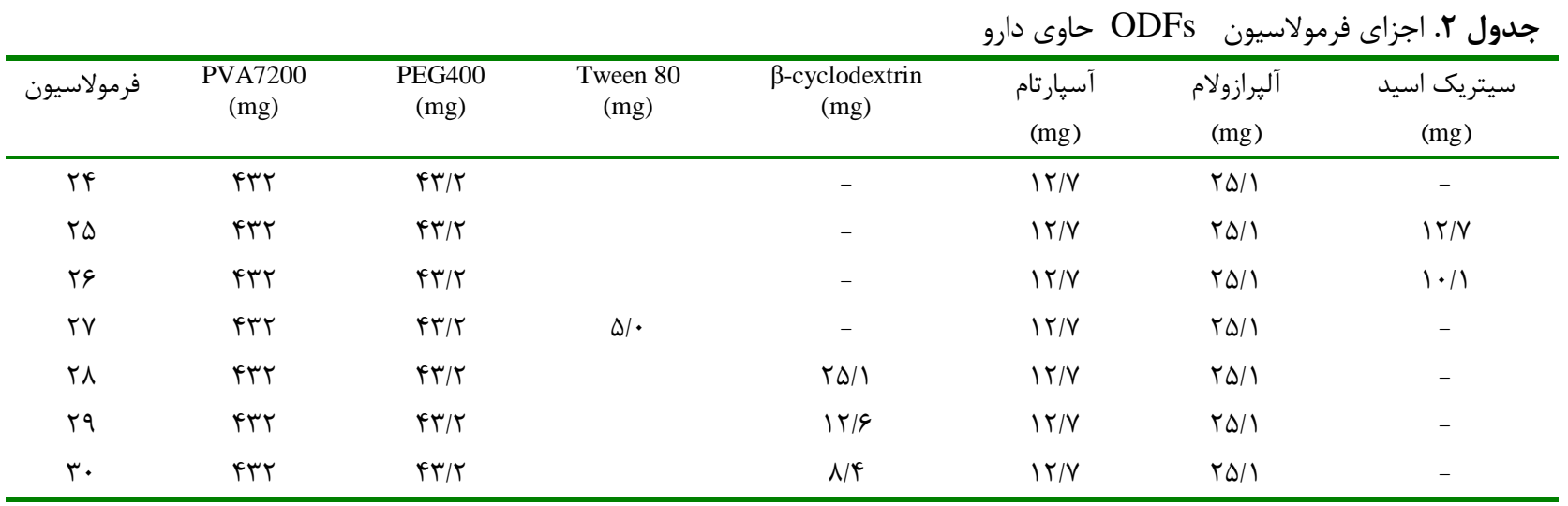

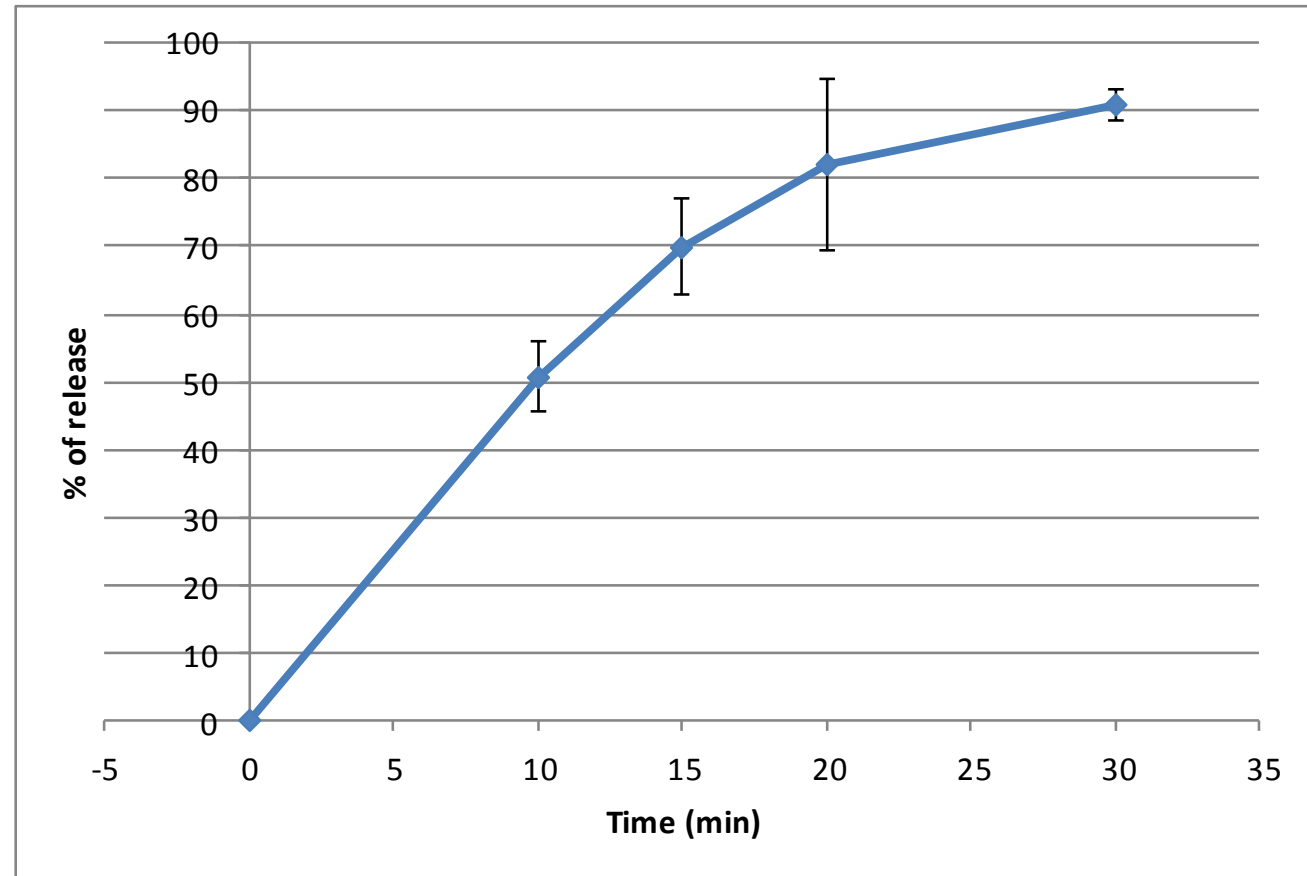

شكل 1. آزادسازى دارو از فرمولاسيون منتخب

$$
\text { تعيين pH فيلمها }
$$

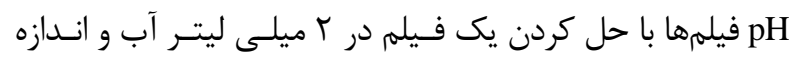

كيرى به كمك pH متر انجام شد (9).

$$
\text { آزمون سرعت حلاليت }
$$

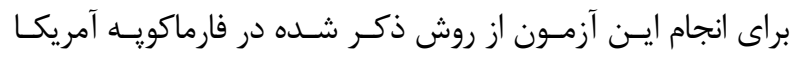

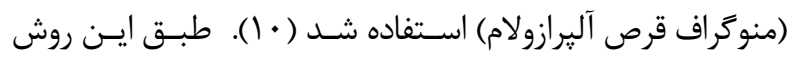

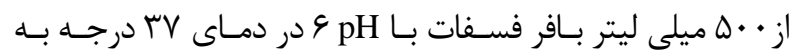

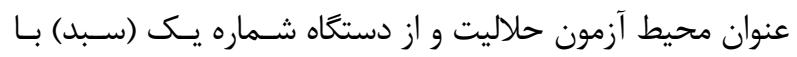

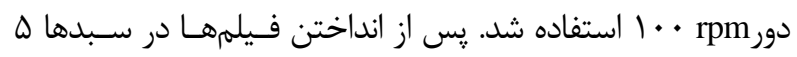

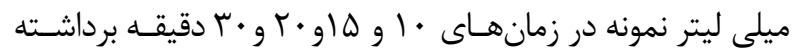

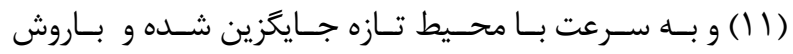

تعبين ضخامت فيلمه ها

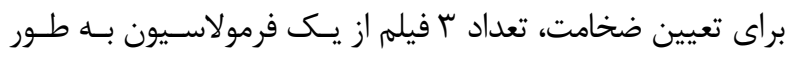

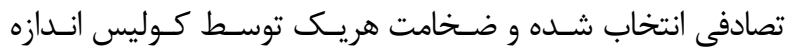
كيرى و ميانكَين ضخامت آن ها و همين طور درصد انحراف معيار

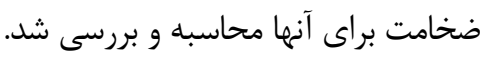

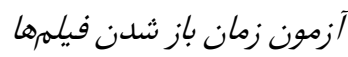

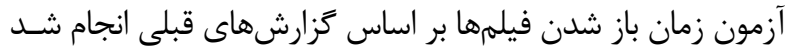

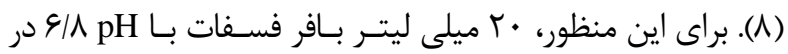

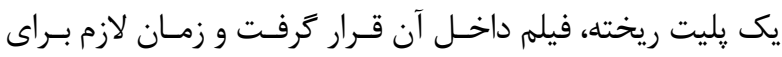

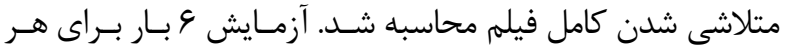

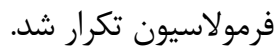


پايه برتر براى اضافه كردن دارو در مرحله بعدى انتخاب

\section{يايه هاى يليمرى داراى دارو}

يس از تهيه فرمولاسيون شماره YF رسوب دارو در قسمت-

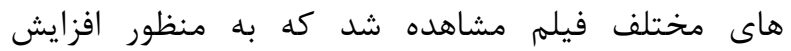

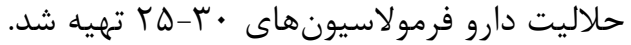
بررسى تاثير pH بر تشكيل فيلم يكنواخت

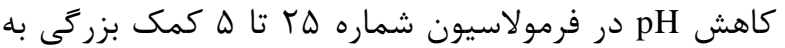
حلاليت آليرازولام كرد، طورى كه فيلمى يكنواخت بدرن

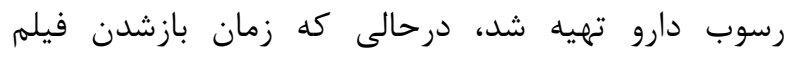
همجنان كمتر از يك دقيقه بود. رسوب دارو كماكان در دران فرمولاسيون شماره عץ با pH 9 مشاهده شد. بررسى تاثير اكسيانتهاى ديكر بر تشكيل فيله يكنواخت افزودن تويين •N هيج كمكى به حل شدن دارو نكرد، اما بتا سيكلودكسترين زمانى كه به ميزان يك سوم دارو به به به

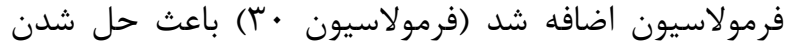

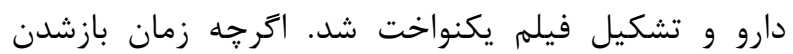
فيلم افزايش يافته، بيشتر از يك دقيقه شد.

\section{آزمون سرعت حلاليت}

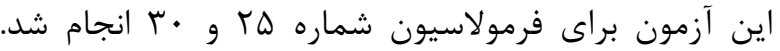

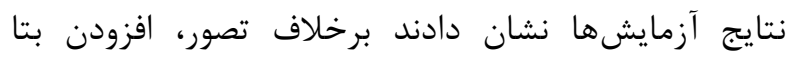

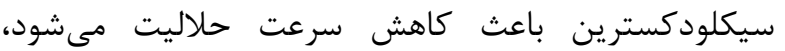

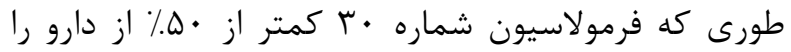

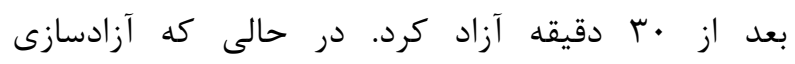

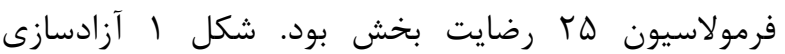
فرمولاسيون شماره فا را نشان مى دهد. تعيين مقدار دارو

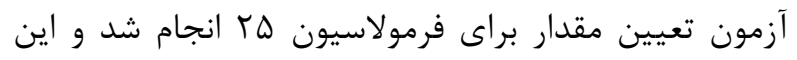

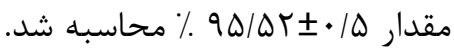

\section{تعيين يكنواختى محتوا}

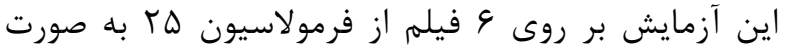

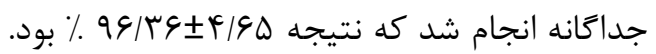

ضخامت فيلم

ضخامت فيلمهاى حاصل از فرمولاسيون

امروزه سيستمهاى دارورسانى مختلفى در بازار دارويى جهان

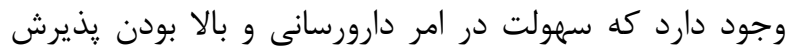

كرومـاتوكرافى مــايع (HPLC High Performance Liquid : (Chromatography ذكر شده در بخش تعيسين مقــدار دارو مـورد آناليز قرار كرفت و نمودار درصد آزادسازى در برابر زمان رسم شد.

آزمون تعيين مقدار دارو

روش HPLC

روش مورد استفاده روش ذكر شده در فارماكويه آمريكا (منــوكراف قرص آليرازولام) بود كه با استفاده از دستگاه يانكلين ساخت كـره دره

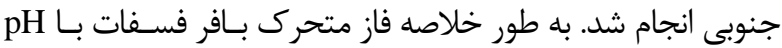

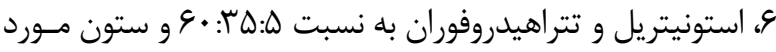
استفاده C8(6/4 mm ×15cm, 5um ساخت شركت هكتور كره جنوبى و طول موج فرا بنفش TDF نـانومتر بـود. فـاز متحسرك بـا سرعت ا ميلى ليتر بر دقيقه از ستون عبور داده شد. حجم تزريق

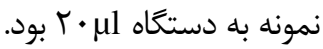
تهيه محلول استاندارد

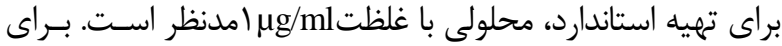

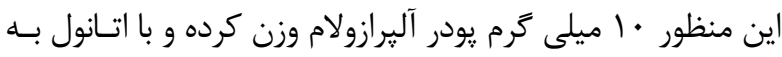

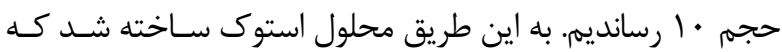

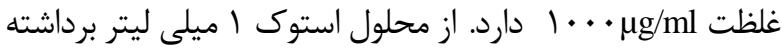

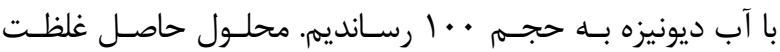

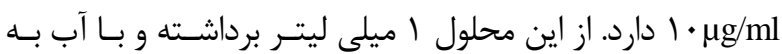

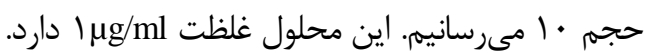
آماده سازى محلول تست آليرازولام

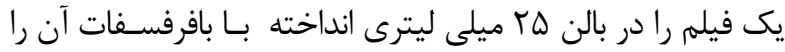
حل كرده ، به حجم مى رسانيم.

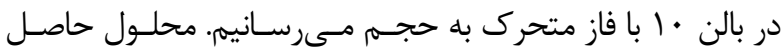

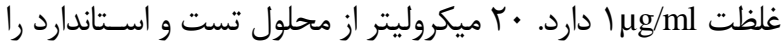

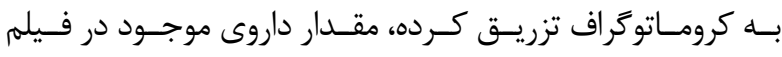
محاسبه مى كردد. آزمايش سه بار انجام شد. آزمون يكنواختى محتوا مقدار آلرازولام شش فيلم از يك فرمولاسيون را به روش مـذكور

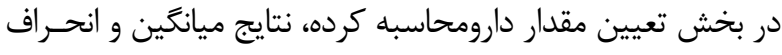
معيار بررسى شد.

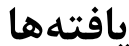

يا يايههاى يليمرى بدون دارو

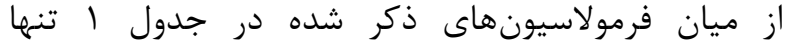
فرمولاسيون شماره ها از نظر زمان باز شدن فيلم (كمتر از

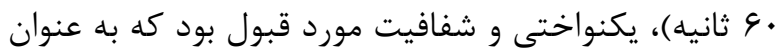


رسوب دارو شد. اين مشكل با كاهش pH فرمولاسيون به ها ها

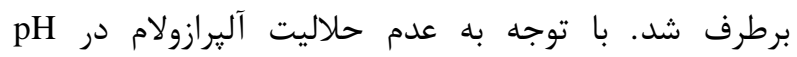

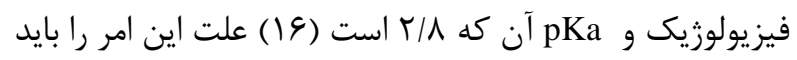

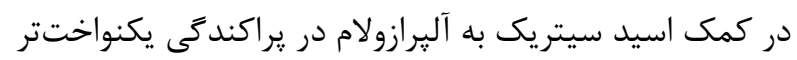

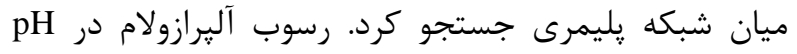

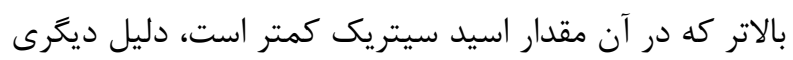

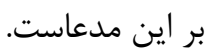

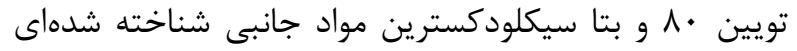

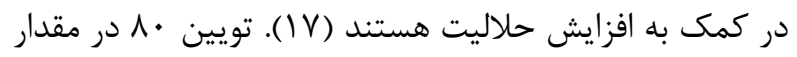

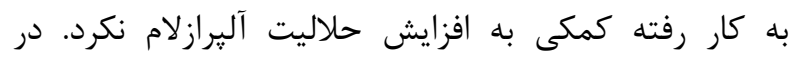

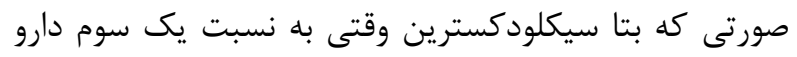
به فرمولاسيون اضافه شد باعث افزايش حلاليت دارو شد،

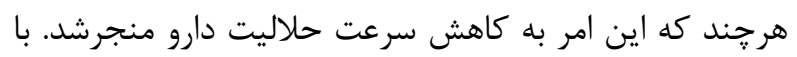

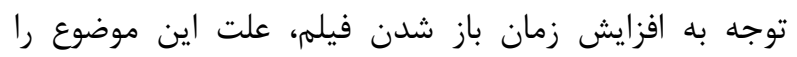

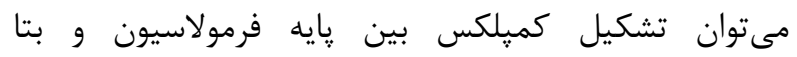

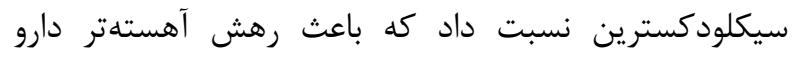

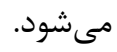
در پايان مىتوان نيتيجه كرفت فرمولاسيون شماره كآ كه

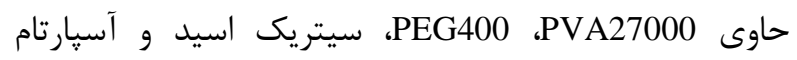

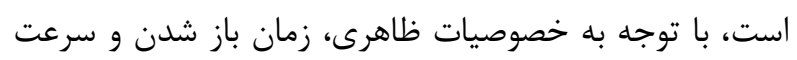
انحلال فرمولاسيون مناسبى است.

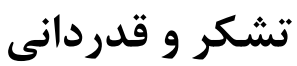

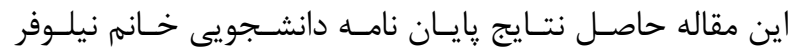

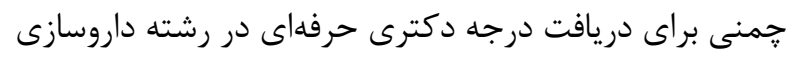

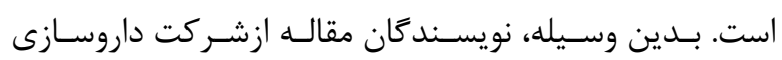

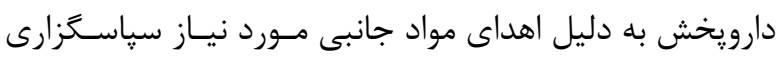
مى كنند.
بيمار از جمله اهداف بسيار مهمم در طراحى آنهاست. در اين

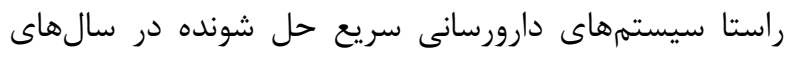

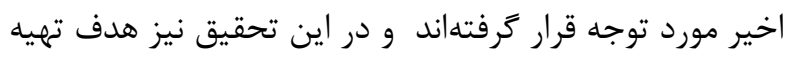

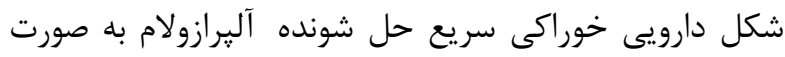

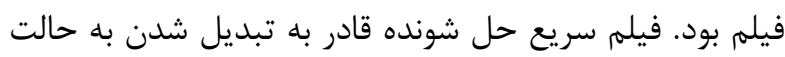

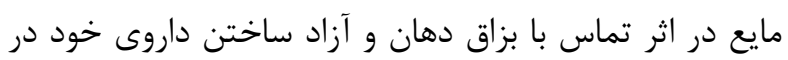

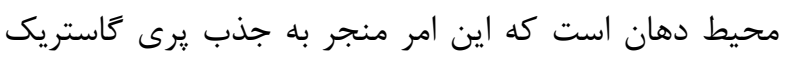

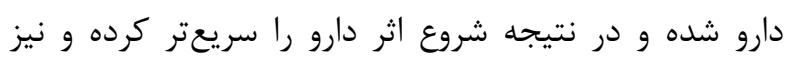

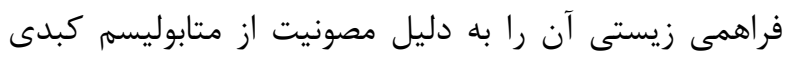

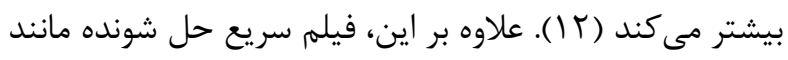

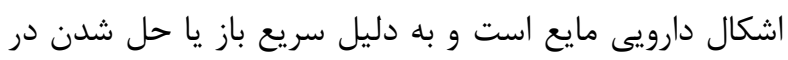

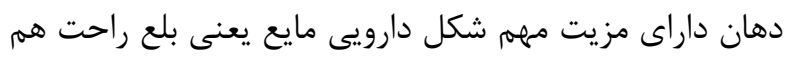

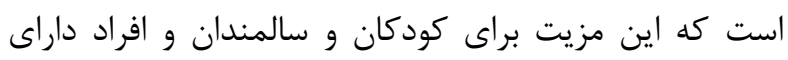

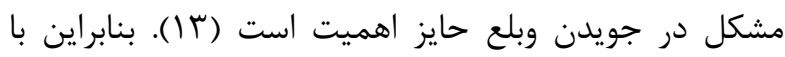

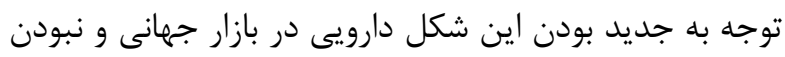

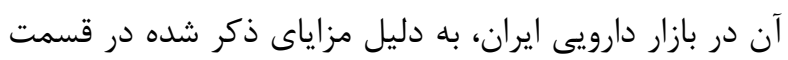

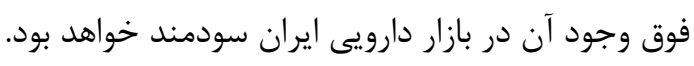

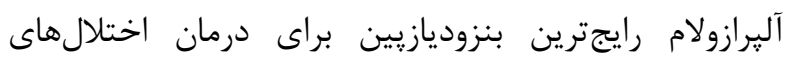

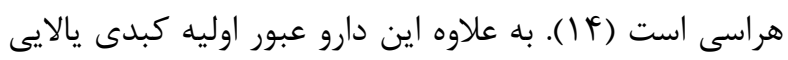

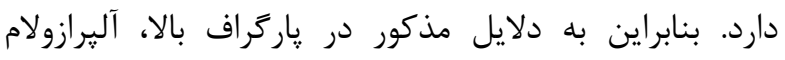

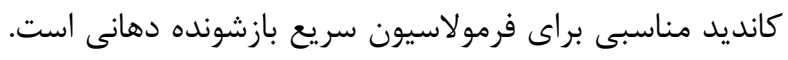

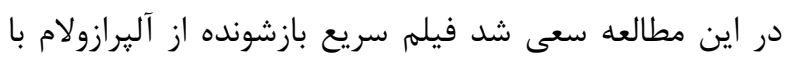

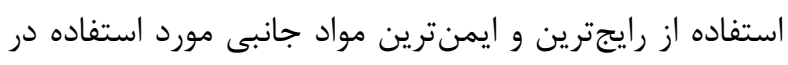

$$
\text { صنايع دارويى ايران تهيه شود. }
$$

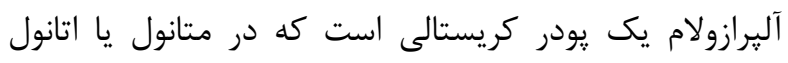

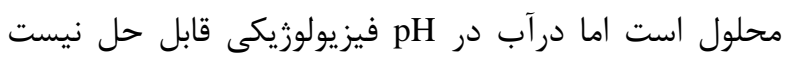

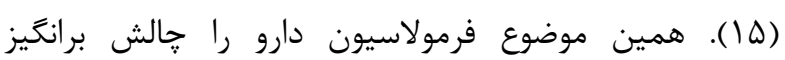

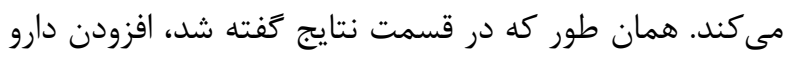

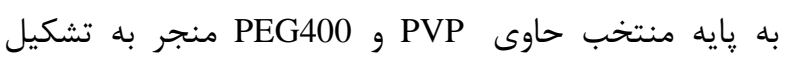

\section{REFERENCES}

1. Goel H, Rai P, Rana V, Tiwary AK. Orally disintegrating systems: innovations in formulation and technology. Recent Pat Drug Deliv Formul 2008;2:258-74.

2. Lee Y, Thapa P, Jeong SH, Woo MH, Choi DH. Formulation Optimization and in Vitro Characterization of Orally Disintegrating Films Using a Factorial Design and Mathematical Modeling for Drug Release. Chem Pharm Bull (Tokyo) 2017;65:166-77.

3. Malallah OS, Garcia CMA, Proctor GB, Forbes B, Royall PG. Buccal drug delivery technologies for patient-centred treatment of radiation-induced xerostomia (dry mouth). Int J Pharm 2018;541:157-66.

4. Isbister GK, O'Regan L, Sibbritt D, Whyte IM. Alprazolam is relatively more toxic than other benzodiazepines in overdose. Br J Clin Pharmacol 2004;58:88-95.

5. Agarwal V, Kommaddi RP, Valli K, Ryder D, Hyde TM, Kleinman JE, et al. Drug metabolism in human brain: high levels of cytochrome P4503A43 in brain and metabolism of anti-anxiety drug alprazolam to its active metabolite. PLoS One 2008;3:e2337 
6. Dinge A, Nagarsenker M. Formulation and evaluation of fast dissolving films for delivery of triclosan to the oral cavity. AAPS PharmSciTech 2008;9:349-56.

7. Samprasit W, Akkaramongkolporn P, Kaomongkolgit R, Opanasopit P. Cyclodextrin-based oral dissolving films formulation of taste-masked meloxicam. Pharm Dev Technol 2017;7:1-10.

8. Liu C, Chang D, Zhang X, Sui H, Kong Y, Zhu R, et al. Oral fast-dissolving films containing lutein nanocrystals for improved bioavailability: formulation development, in vitro and in vivo evaluation. AAPS PharmSciTech 2017;18:2957-64.

9. Garsuch V. Preparation and characterization of fast-dissolving oral films for pediatric use [PhD dissertation]. Düsseldorf: Heinrich-Heine University; 2009.

10. United States Pharmacopeia (USP): 2019. Available from: https://www.uspnf.com/sites/default/files/ usp_pdf/EN/USPNF/revisions/alprazolam-tabs-rb-notice-20190301.pdf

11. Londhe VY, Umalkar KB. Formulation development and evaluation of fast dissolving film of telmisartan. Indian J Pharm Sci 2012;74:122-6.

12. Dahiya M, Saha S, Shahiwala AF. A review on mouth dissolving films. Curr Drug Deliv 2009;6:469-76.

13. Kathpalia H, Gupte A. An introduction to fast dissolving oral thin film drug delivery systems: a review. Curr Drug Deliv 2013;10:667-84.

14. Rabinowitz JD, Lloyd PM, Munzar P, Myers DJ, Cross S, Damani R, et al. Ultra-fast absorption of amorphous pure drug aerosols via deep lung inhalation. J Pharm Sci 2006;95:2438-51.

15. RxList website. https://www.rxlist.com/xanax-drug.htm

16. Oo CY, Kuhn RJ, Desai N, Wright CE, McNamara PJ. Pharmacokinetics in lactating women: prediction of alprazolam transfer into milk. Br J Clin Pharmacol 1995;40:231-6.

17. Pacheco PA, Rodrigues LNC, Ferreira JFS, Gomes ACP, Veríssimo CJ, Louvandini H, et al. Inclusion complex and nanoclusters of cyclodextrin to increase the solubility and efficacy of albendazole. Parasitol Res 2018;117:705-12. 